\title{
Reliability Measurement in A Multi-path Transmission Network Using SMP-BP Algorithm
}

\author{
https://doi.org/10.3991/ijoe.v15i11.10627 \\ Xiaoling Li $\left({ }^{凶}\right)$, Xinwei Zhou \\ Nanchang University Gongqing College, Jiangxi, China
}

6699114749 asina.com

\begin{abstract}
Data security is very important in the multi-path transmission networks (MTN). Efficient data security measurement in MTN is crucial so as to ensure the reliability of data transmission. To this end, this paper presents an improved algorithm using single-single minimal path based back-up path (SSMP-BP), which is designed to ensure the data transmission when the second path is out of work. From the simulation study, the proposed algorithm has the better network reliability compared with existing double minimal path based backup path (DMP-BP) approach. It could be found that, the proposed algorithm uses less back-up paths compared with DMP-BP so that less network resources like nodes are achieved.
\end{abstract}

Keywords-Data Security, Multi-path Transmission, Network, SMPBP, Algorithm, Efficiency

\section{$1 \quad$ Introduction}

Data security is very important in the network where the cloud-based communications and transmissions could be conducted [1]. When carrying out the data transmission, the time for a group of channels or paths without any crossover is less than the time spent on a single path. Multi-path transmission network (MTN) has been widely used in our daily life such as mobile ad hoc networks, cloud-based applications and multi-protocol label switch networks [2-4]. However, MTN is easily influenced by the errors or network fault, which will greatly impact the efficient data transferring and even a system break-down will be happened sometimes.

Efficient data security measurement in the MTN is one of the most significant research areas. It is necessary to measure the efficient data transmission so that the network could be more reliable [5]. One typical approach is using back-up path for ensuring the data security. Data could be transferred in parallel through $k$ paths which are not crossover. That means there is no same links for any two paths. A back-up paths with $k$ possible routes were used for ensuring the efficient data transmission [6]. If there are some faults, the back-up paths could be used. It is with significant to use multiple alternative paths through a network, which can yield a variety of benefits such as fault tolerance, increased bandwidth, or improved security. 
Table 1. A list of symbols is presented in the following in this paper.

\begin{tabular}{|c|l|}
\hline$k$ & Number of paths in the network \\
\hline$P_{i}$ & A path in the network \\
\hline$P_{b i}$ & A back-up path in the network \\
\hline$G$ & A multi-path transmission network (MTN) \\
\hline$N$ & A set of nodes \\
\hline$A$ & A set of linkages \\
\hline$L$ & A set of time delay \\
\hline$C$ & A set of cost for transmission \\
\hline$M_{i}$ & Maximum value of $a_{i} \in A$ \\
\hline$x_{i}$ & Current capacity of $a_{i} \in A$ \\
\hline$m$ & Total number of minimal path \\
\hline$P_{e}$ & A set of minimal path \\
\hline$n_{e}$ & Total number of linkages \\
\hline$S$ & The start node \\
\hline$t$ & The end node \\
\hline$d$ & The data from $S_{\text {to }} t$ \\
\hline$d_{e}$ & Data quantity \\
\hline $\bar{d}_{e}$ & Upper boundary of $d_{e}$ \\
\hline$F\left(d_{e}, P_{e}\right)$ & Total cost \\
\hline$T\left(d_{e}, P_{e}\right)$ & Transmission time \\
\hline$T$ & Time \\
\hline$P_{r}()$ & Reliability \\
\hline$\varsigma, \lambda, o, k, \omega, u$ & Index \\
\hline$I, K, J$ & Index set \\
\hline & \\
\hline
\end{tabular}

Assume that there are $k$ paths $\left(P_{1}, P_{2}, \ldots, P_{i}, \ldots, P_{j}, \ldots, P_{k}\right)$ in the network. If one of them $P_{i}$ is out of service, the back-up path $P_{b i}$ could be used. The current working route will be $\left(P_{1}, P_{2}, \ldots P_{i-1}, P_{b i}, P_{i+1} . . P_{j}, \ldots, P_{k}\right)$. When another path $P_{j}$ $j=1,2, \ldots, i-1, b_{1}, j+1, \ldots, k$ is out of work, based on the $P_{b i}$, another back-up path will be selected $\left(P_{1}, P_{2}, \ldots P_{i-1}, P_{b i}, P_{i+1} . . P_{j-1}, P_{b j}, P_{j+1} \ldots, P_{k}\right)$. In some cases, the working path will be out of service instantly one following another [7], the backup path $P_{b q}$ will be selected based on the previous $P_{b(q-1)}$. In this way, the data security could be ensured. However, how to measure the efficiency of data security under the multi-path transmission system needs to be further studied. 
Some research has been done for the measurement of the reliability. For example, a single minimal path based back-up path (SMP-BP) algorithm was proposed for this purpose [8-10]. SMP-BP uses two individual paths as a working couple where the minimal paths without cross as back-up paths. It supports end-to-end path-based connection restoration in SPR (Shortest Path Restoration), PIR (Partial Information Restoration) and CIR (Complete Information Restoration) networks. If there are any errors, the back-up paths will be triggered [11]. A new path or route will be established with the functions of back-up path in the network.

The shortest path problem usually uses graphs including undirected, directed or mixed models to consider different objective functions such as minimized costs or time. However, some sharing of back-up paths is possible while using the aggregated service bandwidth on each link. It was reported that it is reasonable to increase backup path sharing using aggregated information as with PIR [12]. Low Cost an S-Disjoint (LCSD) paths algorithm is based on a SRLG disjoint active and backup paths for network protection that can be used to avoid the risk sharing with active path $[13,14]$. More precisely, a route for the backup path to minimize the joint path failure probability between the primary and the backup paths will be determined. To demonstrate the feasibility of that, extensive evaluations under both single and double link failure models have been carried out in terms of robustness.

In this paper, an improved algorithm using single-single minimal path based backup path (SSMP-BP) was introduced. SSMP-BP is designed to ensure the data transmission when the second path is out of work. The proposed algorithm uses two noncrossing paths as working routes one of which is non-crossing with the working path. That will be regarded as the back-up. When there are some working path fails, the back-up path will be evoked. A new working path will be created. Thus, there are two paths in the network which can transmit the data at the same time. This algorithm improves the back-up path selection through the non-crossing paths so that the reliability of transmission could be enhanced.

The rest of this paper is organized as follows. Section 2 gives the problem description. Section 3 presents how to use the proposed algorithm to measure the data efficiency. Section 4 gives a simulation case which compares the DMP-BP, SMP-BP, and SSMP-BP. Section 5 concludes this paper.

\section{Problem Description}

Let $G=(N, A, L, C)$ denotes a MTN, $N$ is a set of nodes, $A=\left\{a_{i} \mid i=1,2, . ., n\right\}$ refers to a set of linkages for connecting the nodes. $L=\left\{l_{i} \mid i=1,2, . ., n\right\}$ indicates a set of time delay. In $C=\left\{c_{i} \mid i=1,2, . ., n\right\}, a_{i}$ means the no. $i$ linkage. $l_{i}$ is the transmission delay. $c_{i}$ denotes the cost of the transmission. $M_{i}$ is used to present the maximum value of $a_{i} \cdot x_{i}$ means the current capacity of $a_{i} . X=\left(x_{1}, x_{2}, \ldots, x_{n}\right)$ is a vector showing the status of network capac- 
ity. Let $m$ denotes the total number of minimal path (MP). $P_{e}=\left\{a_{e 1}, a_{e 2}, \ldots, a_{e q}, a_{e n_{e}},\right\}$ refers to no. $e$ MP $e=1,2, \ldots, m \cdot n_{e}$ is the total number of linkages. $a_{e q}$ is the no. $q$ linkage in $P_{e} q=1,2, \ldots, n_{e}$. Let $s$ and $t$ represent the start and end nodes, $d$ means the data from $s$ to $t, d_{e}$ means the data quantity allocated to $P_{e} \cdot \bar{d}_{e}$ is the upper boundary of $d_{e} \cdot B$ and $T$ shows the cost and time constrains respectively.

Some assumptions are made in this paper as follows:

- Every node will not be out of service.

- The capacity for each path/link follows random distribution and they are strictly independent.

- Each path couple contains two non-cross routes.

- The total input flow is equal to the output flow.

Based on the assumptions, let $F\left(d_{e}, P_{e}\right)$ presents the total cost and $T\left(d_{e}, P_{e}\right)$ is the transmission time. The calculation of $F\left(d_{e}, P_{e}\right)$ and $T\left(d_{e}, P_{e}\right)$ will be based on [15], where $\left(d_{e}, c_{e q}\right)$ is the cost of data passing $a_{e q} \cdot \min _{1 \leq q \leq n_{e}} x_{e q}$ is the capacity under the vector $X$ of the network status.

$$
\begin{gathered}
F\left(d_{e}, P_{e}\right)=\sum_{q=1}^{n_{e}}\left(d_{e} g c_{e q}\right) \\
T\left(d_{e}, P_{e}\right)=\sum_{q=1}^{n_{e}} l_{e q}+\left\lceil\frac{d_{e}}{\min _{1 \leq q \leq n_{e}} x_{e q}}\right\rceil
\end{gathered}
$$

If the data from unit $d$ pass $\lambda(\lambda \geq 2)$ paths which are not cross over, $d$ could be divided into $\left(d_{1}, d_{2}, \ldots, d_{\varsigma}, \ldots, d_{\lambda}\right)$. The total transferring cost could be calculated by:

$$
F\left(d,\left(P_{1}, P_{2}, \ldots P_{\varsigma}, P_{\lambda}\right)\right)=\sum_{\varsigma=1}^{\lambda} F\left(d_{\varsigma}, P_{\varsigma}\right)
$$

Where $d=\sum_{\varsigma=1}^{\lambda} d_{\varsigma}, d_{\varsigma} \leq \bar{d}_{\varsigma} . d_{\varsigma}$ is a non-negative integer and $\overline{d_{\varsigma}}$ is the maximum data allocated to $P_{\varsigma}=\left\{a_{\varsigma 1}, \ldots, a_{\varsigma \omega}, \ldots, a_{\varsigma n_{\varsigma}}\right\}$. 


$$
\left.\overline{d_{\varsigma}} \leq\left(T-\sum_{\omega=1}^{n_{\varsigma}} l_{\varsigma \omega}\right) \mathrm{g} \min _{1 \leq \omega \leq n_{\varsigma}} M_{\varsigma \omega}\right\rfloor
$$

Thus, we can get:

$$
\begin{gathered}
T\left(d_{\varsigma}, P_{\varsigma}\right) \leq T \\
F\left(d,\left(P_{1}, P_{2}, \ldots P_{\varsigma}, P_{\lambda}\right)\right) \leq F\left(d_{e}, P_{e}\right)
\end{gathered}
$$

In the multi-path network environment, Internet packet traffic keeps growing as the number of applications and services as well as their bandwidth requirements explode. It then becomes necessary to ensure that network throughput is maximized. In this problem description, dynamic multi-path routing is considered to improve network reliability. Multi-path routing is important for throughput, reliability and security. In multi-path routing, improvements in performance are achieved by utilizing more than one feasible path for more effective network resource utilization. Various research on multipath routing have addressed network redundancy, congestion, and QoS issues using the sensor data at each outgoing network link [11]. The real-time status will be captured by the sensors deployed in the network nodes such as routers, extenders, servers, etc. The time differences could be calculated by the sending and receiving time at different network nodes. For example, one data package $D P$ is sent out by router $A$ in time $T_{1}$ through Internet (TCP/IP). After several time, router $B$ receives the $D P$ at $T_{2}$. The cost of transferring the data could be calculated by $\left(T_{2}-T_{1}\right) \times C_{u}$. $C_{u}$ is the unit cost considering the transmission channel.

\section{Proposed SSMP-BP Algorithm}

\subsection{SMP-BP algorithm}

Define $\left(P_{b}, P_{o}\right)$ and $\left(P_{k}\right) \quad(b, o, k=1,2, \ldots, m, b \neq o \neq k)$ represent the working and back-up paths, the reliability could be calculated by:

$$
P_{r_{S M P-B P}}\left(S \mid P_{b} P_{o}, P_{k}\right)=P_{r}\left(\overline{P_{b}}\right) P_{r}\left(S \mid P_{o} P_{k}\right)+P_{r}\left(\overline{P_{o}}\right) P_{r}\left(S \mid P_{b} P_{k}\right)
$$

The first part $P_{r}\left(\bar{P}_{b}\right) P_{r}\left(S \mid P_{o} P_{k}\right)$ considers possibility of working path that could be in failure and the second part $P_{r}\left(\bar{P}_{o}\right) P_{r}\left(S \mid P_{b} P_{k}\right)$ considers the possibility of back-up path which could be working after the failure of current working path so that the total reliability of the network could be optimized. $P_{r}$ is the probability of failure 
at node $r$ at the condition of current working path failure $P_{r}\left(\overline{P_{b}}\right)$ and back-up path working $P_{r}\left(\overline{P_{o}}\right)$. And $S \mid P_{o} P_{k}$ is the conditional probability of the survival of current working path when it is failed.

Assume that at least one of the path will be out of service, then $P_{r}\left(\overline{P_{w}}\right)$ will be

$$
P_{r}\left(\overline{P_{w}}\right)=\left(1-\prod_{r: a_{r} \in P_{\omega}} P_{r}\left(x_{r} \geq 1\right)\right)
$$

The low boundary of the capacity vector $(d, T, B, M P)-L B P S$ will be considered under the time and cost constraints. The measurement of $P_{r}\left(S \mid P_{i} P_{j}\right)$ and $P_{r}\left(S \mid P_{k}\right) \quad$ will $\quad$ be based on $\left(d, T, B,\left(p_{i}, p_{j}\right)\right)-L B P S \quad$ and $\left(d, T, B,\left(P_{k}\right)\right)-L B P \quad$. Let $P_{i}=\left\{a_{i 1}, \ldots, a_{i \alpha}, \ldots, a_{i n_{i}}\right\}$, $P_{j}=\left\{a_{j 1}, \ldots, a_{j \beta}, \ldots, a_{j n_{j}}\right\}, X X$ and $X X_{\text {min }}$ are used for keeping the vector candidates $\left(d, T, B,\left(p_{i}, p_{j}\right)\right)-L B P s$. Set $I$ and $K$ are used for keeping the indexes of non- $\left(d, T, B,\left(p_{i}, p_{j}\right)\right)-L B P S$ and $\left(d, T, B,\left(p_{i}, p_{j}\right)\right)-L B P S . J$ is used to keep the index of $\left(d, T, B,\left(p_{i}, p_{j}\right)\right)-L B P s$. Let $h_{v}$ denotes the total number of $\left(d, T, B,\left(p_{i}, p_{j}\right)\right)-L B P S$ in $J$, then, $\left(d, T, B,\left(p_{i}, p_{j}\right)\right)-X_{v}$ is the status vector of a network capacity. $P_{r}\left(S \mid P_{i} P_{j}\right)$ could be expressed as follows:

$$
P_{r}\left(S \mid P_{i} P_{j}\right)=P_{r}\left\{\bigcup_{v=1}^{h_{v}} B_{v}\right\}
$$

Where $B_{v}=\left\{X \mid X \geq X_{v}\right\}$.

\subsection{SMP-BP characteristics}

SMP-BP has some characteristics, which are defined as follows.

- The prerequisite of the successful data transferring event for a single path $\left(P_{k}\right)$ is the maximum capacity should not be less than required capacity $\overline{d_{k}} \geq d$.

- The prerequisite of the successful data transferring event for two paths $\left(P_{i}, P_{j}\right)$ is the sum of both maximum capacity should not be less than the required capacity, $\bar{d}_{i}+\bar{d}_{j} \geq d$. 
Data could be transmitted through two ways: single or two path, by comparing the network reliability $P_{r}\left(S \mid P_{f}\right)$ and $P_{r}\left(S \mid P_{f} P_{g}\right)$, some conclusions could be made: $P_{r}\left(S \mid P_{f} P_{g}\right) \geq P_{r}\left(S \mid P_{f}\right)$

If one path is out of service, we can get

$$
P_{r_{S M P-B P}}\left(S \mid P_{b} P_{o}, P_{k}\right) \geq P_{r_{D M P-B P}}\left(S \mid P_{b} P_{o}, P_{i} P_{j}\right),
$$

$b, o, k, i, j=1,2, \ldots, m ; b \neq o \neq k, b \neq o \neq i \neq j$. That means the reliability of SMP-BP is better than DMP-BP because SMP-BP uses less back-up paths achieving the more efficient data transmission so as to save the network resources.

\subsection{SSMP-BP model}

Let $P_{k}$ and $P_{k k}$ present two back-up paths. $\left\langle P_{k}, P_{k k}\right\rangle$ reveals the orders of these two paths. If the second path is out of service, the reliability could be measured by:

$$
\begin{aligned}
& P_{r_{S S M P-B P}}\left(S \mid P_{b} P_{o},<P_{k}, P_{k k}>\right)=P_{r}\left(\overline{P_{b}}\right)\left[P_{r}\left(\overline{P_{o}}\right) P_{r}\left(S \mid P_{k} P_{k k}\right)+P_{r}\left(\overline{P_{k}}\right) P_{r}\left(S \mid P_{o} P_{k k}\right)\right] \\
+ & P_{r}\left(\overline{P_{o}}\right)\left[P_{r}\left(\overline{P_{b}}\right) P_{r}\left(S \mid P_{k} P_{k k}\right)+P_{r}\left(\overline{P_{k}}\right) P_{r}\left(S \mid P_{b} P_{k k}\right)\right] \\
= & 2 P_{r}\left(\overline{P_{b}}\right) P_{r}\left(\overline{P_{o}}\right) P_{r}\left(S \mid P_{k} P_{k k}\right)+P_{r}\left(\overline{P_{k}}\right) P_{r_{S M P-B P}}\left(S \mid P_{b} P_{O}, P_{k k}\right)
\end{aligned}
$$

Formula (10) considers two back-up paths in the network system. $P_{r}\left(\overline{P_{b}}\right)$ is the probability of network failure of current working path. $P_{r}\left(\overline{P_{o}}\right) P_{r}\left(S \mid P_{k} P_{k k}\right)$ is the working probability of the first back-up path and $P_{r}\left(\bar{P}_{k}\right) P_{r}\left(S \mid P_{o} P_{k k}\right)$ represents the second one which has the probability for replacing the first one. $P_{r}\left(\overline{P_{o}}\right)$ indicates the probability of failure of any back-up path since two back-up paths are considered. $P_{r}\left(\overline{P_{b}}\right) P_{r}\left(S \mid P_{k} P_{k k}\right)+P_{r}\left(\bar{P}_{k}\right) P_{r}\left(S \mid P_{b} P_{k k}\right)$ is the alternative back-up path selection if one of them is failed.

Under this case, if the back-up path couple is $\left(P_{k}, P_{k k}\right)$, based on the DMP-BP approach, the network reliability is:

$$
P_{r_{D M P-B P}^{\prime}}^{\prime}\left(S \mid P_{b} P_{o}, P_{k} P_{k k}\right)=P_{r}\left(\overline{P_{b}}\right) P_{r}\left(\overline{P_{o}}\right) P_{r}\left(S \mid P_{k} P_{k k}\right)
$$

Based on (10) and (11), it could be observed that under the failure of second backup path,

$$
P_{r_{S S M P-B P}}\left(S \mid P_{b} P_{o},<P_{k}, P_{k k}>\right) \geq P_{r_{D M P-B P}^{\prime}}^{\prime}\left(S \mid P_{b} P_{o}, P_{k} P_{k k}\right)
$$


$b, o, k, k k=1,2, \ldots, m ; b \neq o \neq k \neq k k$.

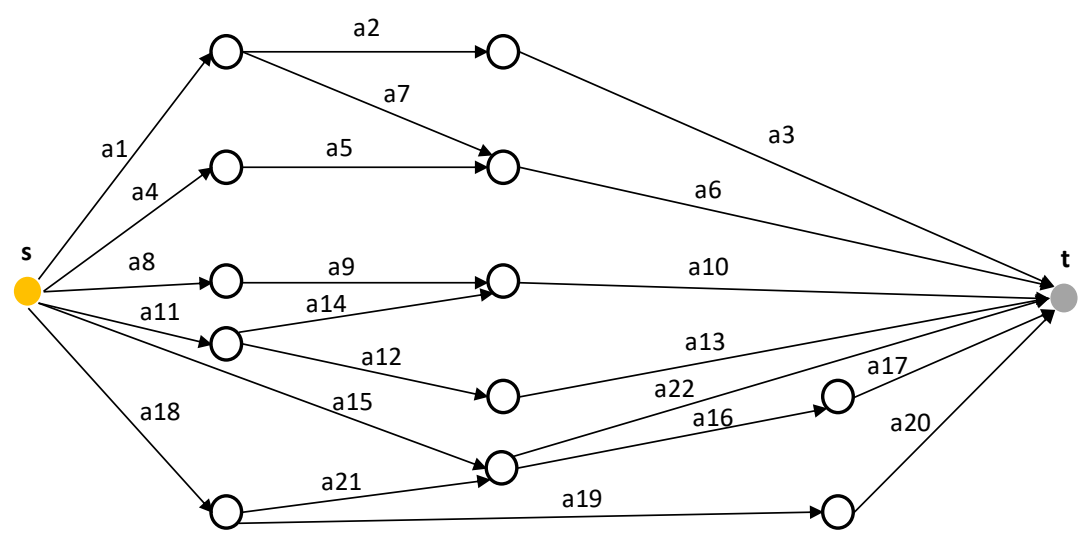

Fig. 1. A multi-path transmission network

\section{Simulation Study}

This section reports on a simulation study based on the following network structure as shown in Fig. 1. Some parameters are set as follows so that the proposed model could be evaluated by comparing with other approaches. $d=200, T=13$, $B=2000$. Assume the working paths couples are $P_{1}=\left(a_{1}, a_{2}, a_{3}\right)$ and $P_{2}=\left(a_{4}, a_{5}, a_{6}\right)$. From Figure 1 , the paths with non-crossing relation with $P_{1}$ and $P_{2}$ are $P_{3}=\left(a_{8}, a_{9}, a_{10}\right), P_{4}=\left(a_{11}, a_{12}, a_{13}\right), P_{5}=\left(a_{15}, a_{16}, a_{17}\right)$, $P_{6}=\left(a_{18}, a_{19}, a_{20}\right), P_{7}=\left(a_{10}, a_{11}, a_{14}\right), P_{8}=\left(a_{15}, a_{22}\right), P_{9}=\left(a_{18}, a_{21}, a_{22}\right)$ and $P_{10}=\left(a_{16}, a_{17}, a_{18}, a_{21}\right)$.

The data is from [16], in DMP-MP algorithm, when the working path is $\left(P_{1}, P_{2}\right)$, the reliability for the back-up path is listed in Table 1.

Table 2. Reliability list

\begin{tabular}{|c|c|c|c|c|c|c|c|}
\hline $\boldsymbol{i}, \boldsymbol{j}$ & $P_{r}\left(S \mid P_{i} P_{j}\right)$ & $\boldsymbol{i}, \boldsymbol{j}$ & $P_{r}\left(S \mid P_{i} P_{j}\right)$ & $\boldsymbol{i}, \boldsymbol{j}$ & $P_{r}\left(S \mid P_{i} P_{j}\right)$ & $i, j$ & $P_{r}\left(S \mid P_{i} P_{j}\right)$ \\
\hline 3,4 & 0.78 & 3,10 & 0.40 & 4,10 & 0.70 & 6,8 & 0.55 \\
\hline 3,5 & 0.77 & 4,5 & 0.89 & 5,6, & 0.78 & 7,8 & 0 \\
\hline 3,6 & 0.70 & 4,6 & 0.79 & 5,7 & 0.71 & 7,9 & 0 \\
\hline 3,8 & 0.52 & 4,8 & 0.72 & 5,9 & 0.61 & 7,10 & 0 \\
\hline 3,9 & 0.47 & 4,9 & 0.65 & 6,7 & 0.61 & 8,10 & 0 \\
\hline
\end{tabular}


The maximum value from Table 1 is $P_{r}\left(S \mid P_{4} P_{5}\right)=0.89$. Thus, $\left(P_{4}, P_{5}\right)$ is the best back-up path couple. In this simulation study, we use it as the back-up path and the comparison of SSMP-BP and DMP-BP approaches is conducted.

Let $P_{4}$ present the back-up path in SSMP-BP, there are two path failure events with the out of service from $P_{1}$ and $P_{2}$. When $P_{1}$ fails, $P_{4}$ will be triggered to replace $P_{1}$ for sending the data with the help from $P_{2}$. In this situation, $(200,13,2000$, $\left.\left(P_{2}, P_{4}\right)\right)-L B P S: X_{1}$ and $X_{7}$ will be occurred twice. $P_{r}\left(S \mid P_{2} P_{4}\right)=0.71$. When $P_{2}$ fails, $\left(200,13,2000,\left(P_{1}, P_{4}\right)\right)-L B P S: X_{1}, X_{7}, X_{14}$ and $X_{21}$ will be occurred four times. $P_{r}\left(S \mid P_{1} P_{4}\right)=0.89$.

Under the constraints of $T$ and $B$, for the data transmission through $\left(P_{1}\right)$, there is one $\left(200,13,2000,\left(P_{1}\right)\right)-L B P: X_{L B P}=(50,50,40,0,0,0,0,0,0,0,0,0,0,0$, $0,0,0,0,0,0,0,0)$. Thus, we can get $P_{r}\left(S \mid P_{1}\right)=0.58$. Since $\overline{d_{2}}=120 \leq d$, then $P_{r}\left(S \mid P_{2}\right)=0$, according to Equation (8), we can get $P_{r}\left(\bar{P}_{1}\right)=0.14$ and $P_{r}\left(\overline{P_{2}}\right)=0.14$. Based on the Equation (7) and (10), we can get the following results under path failure case using $P_{4}$ as a back-up path:

$$
\begin{aligned}
& P_{r_{D N P}-B P}\left(S \mid P_{1} P_{2}, P_{4} P_{5}\right)=P_{r}\left(\bar{P}_{1}\right) P_{r}\left(S \mid P_{2}\right)+P_{r}\left(\bar{P}_{2}\right) P_{r}\left(S \mid P_{1}\right)=0.14 \times 0+0.14 \times 0.58=0.08 \\
& P_{r_{S S M P-B P}}\left(S \mid P_{1} P_{2}, P_{4}\right)=P_{r}\left(\bar{P}_{1}\right) P_{r}\left(S \mid P_{2} P_{4}\right)+P_{r}\left(\bar{P}_{2}\right) P_{r}\left(S \mid P_{1} P_{4}\right)=0.14 \times 0.71+0.14 \times 0.89=0.23 \\
& P_{r_{S S M P-B P}}\left(S \mid P_{1} P_{2}, P_{4}\right) P_{-} P_{r_{D M P-B P}}\left(S \mid P_{1} P_{2}, P_{4} P_{5}\right)_{=0.15}
\end{aligned}
$$

Similarly, if we use $P_{5}$ as the back-up path, we can get:

$$
\begin{aligned}
& P_{r}\left(S \mid P_{1} P_{5}\right)=0.88, P_{r}\left(S \mid P_{2} P_{5}\right)=0.70 \\
& P_{r_{\text {SSIP }-B P}}\left(S \mid P_{1} P_{2}, P_{5}\right)=P_{r}\left(\bar{P}_{1}\right) P_{r}\left(S \mid P_{2} P_{5}\right)+P_{r}\left(\bar{P}_{2}\right) P_{r}\left(S \mid P_{1} P_{5}\right)=0.14 \times 0.70+0.14 \times 0.88=0.23 \\
& P_{r_{\text {SSUP }-B P}}\left(S \mid P_{1} P_{2}, P_{5}\right)-P_{r_{\text {DMP }-B P}}\left(S \mid P_{1} P_{2}, P_{4} P_{5}\right)=0.15
\end{aligned}
$$

It could be concluded that, using $P_{4}, P_{5}$ as the back-up paths, the network efficiency or reliability could be improved by 0.14 (14\%). Using the proposed algorithm, under the failure of the second path, since $P_{4}$ is serving as a working path, the possi- 
bility is $P_{1}, P_{2}$ or $P_{4}$. According to the rule that data should be transmitted by noncrossing paths, the following paths will be available $P_{3}, P_{5}, P_{6}, P_{8}, P_{9}$ and $P_{10}$. When the main path is $\left(P_{1}, P_{2}\right)$, the first and second back-up paths are $P_{4}$ and $P_{k k}$ respectively. Based on equation (10), $P_{r_{\text {SSMP } B P}}\left(S \mid P_{1} P_{2},<P_{4}, P_{k k}>\right)=2 P_{r}\left(\bar{P}_{1}\right) P_{r}\left(\overline{P_{2}}\right)$ $P_{r}\left(S \mid P_{4} P_{k k}\right)+P_{r}\left(\bar{P}_{4}\right) P_{r_{\text {SMP }-B P}}\left(S \mid P_{1} P_{2}, P_{k k}\right)$. Thus, we can get the results from Table 2.

Table 3. Reliability list

\begin{tabular}{|l|c|c|c|c|}
\hline$P_{k k}$ & $P_{r}\left(S \mid P_{1} P_{k k}\right)$ & $P_{r}\left(S \mid P_{2} P_{k k}\right)$ & $P_{r}\left(S \mid P_{4} P_{k k}\right)$ & $P_{r_{S S M P-B P}}\left(S \mid P_{1} P_{2},<P_{4}, P_{k k}>\right)$ \\
\hline$P_{3}$ & 0.78 & 0.51 & 0.78 & 0.06 \\
\hline$P_{5}$ & 0.88 & 0.70 & 0.89 & 0.07 \\
\hline$P_{6}$ & 0.78 & 0.52 & 0.79 & 0.06 \\
\hline$P_{8}$ & 0.68 & 0 & 0.72 & 0.04 \\
\hline$P_{9}$ & 0.67 & 0 & 0.65 & 0.04 \\
\hline$P_{10}$ & 0.58 & 0 & 0.70 & 0.04 \\
\hline
\end{tabular}

From Table 2, it could be observed that $P_{r}\left(\overline{P_{4}}\right)=0.14$. Due to the maximum value of $P_{r_{\text {SSMP } B P}}\left(S \mid P_{1} P_{2},<P_{4}, P_{5}>\right)=0.07$ when $k k=3,5,6,8,9,10, P_{5}$ is selected to be the second back-up path. Thus, the first and second back-up paths will be $P_{4}$ and $P_{5}$. The network reliability will be $P_{r_{\text {SSUP }-B P}}\left(S \mid P_{1} P_{2},<P_{4}, P_{5}>\right)=0.07$. When the back-up path couple is $\left(P_{4}, P_{5}\right)$, when the path couple is out of service, using CMPBP algorithm, the reliability is $P_{r_{D M P-B P}^{\prime}}\left(S \mid P_{1} P_{2}, P_{4} P_{5}\right)=0.02$. While, using the proposed algorithm, we can get:

$$
P_{r_{S S M P-B P}}\left(S \mid P_{1} P_{2},<P_{4}, P_{5}>\right)-P_{r_{D M P-B P}^{\prime}}\left(S \mid P_{1} P_{2}, P_{4} P_{5}\right)=0.05
$$

That implies when the second path is failed, the network reliability will be increased by $0.05(5 \%)$ using the proposed algorithm if $P_{4}$ and $P_{5}$ are used as the back-up paths. For the small scale network, traverse algorithms could be used for finding out the best back-up paths $[17,18]$. However, for intermediate or large scale networks, these algorithms are not able to work properly [19]. The proposed SSMPBP approach could be extended to a network with $u(u \geq 2)$ paths. If there are some working paths $\left(P_{1}, P_{2}, \ldots, P_{i}, \ldots, P_{u}\right), P_{\tau}$ is the back-up path, where $P_{\tau}$ and $P_{i}$ are non-crossing paths $i=1,2, \ldots, u$. Based on the proposed approach, the network reliability could be calculated by: 


$$
\begin{aligned}
P_{r_{G}} & \left(S \mid P_{1} P_{2} \ldots P_{u}, P_{\tau}\right)=P_{r}\left(\overline{P_{1}}\right) P_{r}\left(S \mid P_{2} \ldots P_{u}, P_{\tau}\right) \\
& +P_{r}\left(\overline{P_{2}}\right) P_{r}\left(S \mid P_{1} P_{3} \ldots P_{u}, P_{\tau}\right)+\ldots \\
& +P_{r}\left(\overline{P_{u}}\right) P_{r}\left(S \mid P_{1} P_{2} \ldots P_{u-1}, P_{\tau}\right)
\end{aligned}
$$

\section{Conclusion}

This paper introduces a SMP-BP algorithm to improve the network reliability under the situation of one transmission path failure. SMP-BP uses two non-crossing paths as working routes. And one path which is non-crossing with the working path will be used as the back-up. When a working path fails, the back-up path will be evoked and a new working path will be created with another working path. Thus, there are two paths in the network which can transmit the data at the same time. From the simulation study, the proposed algorithm has a better network reliability compared with existing DMP-BP approach. It could be found that, the proposed algorithm uses less back-up paths compared with DMP-BP so that less network resources like nodes are achieved.

Future research directions will be carried out in the following aspects. Firstly, the network disturbances are ignored in this research. Some disturbances such as power failure could be considered so that some probability theory could be integrated into this algorithm. Secondly, simulation study is only conducted. A testing scenario will be created in the future to evaluate the feasibility and practicality of this proposed algorithm.

\section{Acknowledgement}

This paper is supported by the project "Study of the web data security measurement under cloud computing" (No. 151513) from Science and Technology Fund in Department of Education, Jiangxi, China.

\section{$7 \quad$ References}

[1] Liew, S.-Y., C.-K. Tan, M.-L. Gan and H. G. Goh (2018). A Fast, Adaptive, and EnergyEfficient Data Collection Protocol in Multi-Channel-Multi-Path Wireless Sensor Networks. IEEE Computational Intelligence Magazine 13(1): 30-40. https://doi.org/10.1109 /mci.2017.2773800

[2] Arianpoo, N., I. Aydin and V. C. Leung (2017). Network Coding as a Performance Booster for Concurrent Multi-Path Transfer of Data in Multi-Hop Wireless Networks. IEEE Transactions on Mobile Computing 16(4): 1047-1058. https://doi.org/10.1109/tmc.2016.258 $\underline{5106}$

[3] Kumar, R., D. Kumar and D. Kumar (2017). EACO and FABC to multi-path data transmission in wireless sensor networks. IET Communications 11(4): 522-530. https://doi.org/ $\underline{10.1049 / \text { iet-com.2016.0859 }}$ 
[4] Usman, M., N. Yang, M. A. Jan, X. He, M. Xu and K.-M. Lam (2018). A joint framework for QoS and QoE for video transmission over wireless multimedia sensor networks. IEEE Transactions on Mobile Computing 17(4): 746-759. https://doi.org/10.1109/tmc.2017. $\underline{2739744}$

[5] Ge, X., L. Pan, Q. Li, G. Mao and S. Tu (2017). "Multipath cooperative communications networks for augmented and virtual reality transmission." IEEE Transactions on Multimedia 19(10): 2345-2358. https://doi.org/10.1109/tmm.2017.2733461

[6] Kotian, R., G. Exarchakos, S. Stavros and A. Liotta (2017). "Impact of transmission power control in multi-hop networks." Future Generation Computer Systems 75: 94-107. https://doi.org/10.1016/j.future.2016.10.010

[7] Liu, A., Q. Zhang, Z. Li, Y.-j. Choi, J. Li and N. Komuro (2017). "A green and reliable communication modeling for industrial internet of things." Computers \& Electrical Engineering 58: 364-381. https://doi.org/10.1016/j.compeleceng.2016.09.005

[8] Zhang, H., P. Dong, S. Yu and J. Song (2017). "A scalable and smart hierarchical wireless communication architecture based on network/user separation." IEEE Wireless Communications 24(1): 18-24. https://doi.org/10.1109/mwc.2017.1600135wc

[9] Jang, S., S.-G. Kang and S.-G. Yoon (2017). "CAMR: Congestion-Aware Multi-Path Routing Protocol for Wireless Mesh Networks." Journal of Electrical Engineering \& Technology 12(1): 411-419. https://doi.org/10.5370/jeet.2017.12.1.411

[10] Casoni, M., C. A. Grazia and M. Klapez (2017). "SDN-Based Resource Pooling to Provide Transparent Multi-Path Communications." IEEE Communications Magazine 55(12): 172178. https://doi.org/10.1109/mcom.2017.1601214

[11] Kim, W. and W. Yoon (2017). "Multi-constrained Max-Min Fair Resource Allocation in Multi-channel Wireless Sensor Networks." Wireless Personal Communications 97(4): 5747-5765. https://doi.org/10.1007/s11277-017-4807-x

[12] Zhong, R. Y., Q. Dai, T. Qu, G. Hu and G. Q. Huang (2013). "RFID-enabled real-time manufacturing execution system for mass-customization production." Robotics and Computer-Integrated Manufacturing 29(2): 283-292. https://doi.org/10.1007/s11277-017-4807$\underline{x}$

[13] Holfeld, B., D. Wieruch, T. Wirth, L. Thiele, S. A. Ashraf, J. Huschke, I. Aktas and J. Ansari (2016). "Wireless Communication for Factory Automation: an opportunity for LTE and 5G systems." IEEE Communications Magazine 54(6): 36-43. https://doi.org/10.1109 /mcom.2016.7497764

[14] Sundaresan, S., R. Doss, W. Zhou and S. Piramuthu (2015). "Secure ownership transfer for multi-tag multi-owner passive RFID environment with individual-owner-privacy." Computer Communications 55: 112-124. https://doi.org/10.1016/j.comcom.2014.08.015

[15] Wan, Z., S. Liu, W. Ni and Z. Xu (2018). "An energy-efficient multi-level adaptive clustering routing algorithm for underwater wireless sensor networks." Cluster Computing: 110. https://doi.org/10.1007/s10586-018-2376-8

[16] Lin, Y.-K. (2010). "Spare routing reliability for a stochastic flow network through two minimal paths under budget constraint." IEEE transactions on reliability 59(1): 2-10. https://doi.org/10.1109/tr.2010.2040765

[17] Zhu, L., R. Wang and H. Yang (2017). "Multi-path Data Distribution Mechanism Based on RPL for Energy Consumption and Time Delay." Information 8(4): 124. https://doi.org/ 10.3390/info8040124

[18] Iqbal, A., S. Shah and M. Amir (2017). "Adaptive Investigating Universal Filtered MultiCarrier (UFMC) Performance Analysis in 5G Cognitive Radio Based Sensor Network (CSNs)." International Journal of Engineering Works 4(1): 5-9. 
[19] Ahmed, E., M. Imran, M. Guizani, A. Rayes, J. Lloret, G. Han and W. Guibene (2017). "Enabling mobile and wireless technologies for smart cities." IEEE Communications Magazine 55(1): 74-75. https://doi.org/10.1109/mcom.2017.7823341

\section{Authors}

Xiaoling Li is an Associate Professor and Senior Engineer in the Nanchang University Gongqing College, Jiujiang, Jiangxi, China. Her research interests include data security measurement and computer networks. She has published several papers in international journals and conferences.

Xinwei Zhou is an Lecturer and Engineer in Computer Network from the Nanchang University Gongqing College, Jiujiang, Jiangxi,China. His research focuses on computer networks and network reliability. He has published several papers in international journals and conferences.

Article submitted 2019-04-08. Resubmitted 2019-05-16. Final acceptance 2019-05-09. Final version published as submitted by the authors. 